\title{
Génération de profils de raies X et UV dans les plasmas chauds
}

\author{
A. Calisti, L. Godbert, R. Stamm et B. Talin
}

Equipe "Diagnostics dans les Gaz et les Plasmas", URA 773, Universite de Provence, Centre Saint Jérôme, 13397 Marseille Cedex 13, France

\begin{abstract}
A numerical code capable of generating the spectral profiles of lines radiated by plasmas has been developed. Electronic and ionic Stark broadening and ion motion effects are included. The numerical methods are particularly designed to reduce the computer time necessary to calculate complex atomic structures on work stations.
\end{abstract}

Le code de calcul que nous avons développé permet de générer des profils de raies émises par des plasmas. Il peut être utilisé pour des émetteurs multi-électroniques pour des conditions de plasma très larges, avec des temps de calcul compatibles avec les stations de travail actuelles. Le code est optimisé pour le calcul des spectres de raies dont la forme est dominée par l'effet Stark dû aux particules chargées en mouvement autour des émetteurs ioniques.

Le programme utilise des résultats fournis par d'autres codes:

-physique atomique détaillée pour le calcul de l'effet Stark

-populations des niveaux issues de calculs de cinétique de population hors ETL

-données statistiques sur le microchamp provenant de programmes spécialisés (APEX) et de simulations numériques.

C'est un des éléments d'un programme de synthèse de spectres, orienté vers l'étude des plasmas denses et chauds, en cours de développement dans le cadre d'une collaboration internationale.

Le calcul des spectres de raies dans les plasmas denses et chauds présente un double intérêt: plasmas

-accompagner et anticiper les études de spectroscopie dans ces

-répondre à un besoin de synthèse de spectres pour réaliser des simulations détaillées de transfert radiatif.

Dans les plasmas denses et chauds, comme dans les plasmas produits par laser $\left(\mathrm{N}_{\mathrm{e}}=10^{20} \mathrm{~cm}^{-3}, \mathrm{~T}_{\mathrm{e}}=10^{6} \mathrm{~K}\right)$, les intérieurs d'étoiles ou les plasmas d'implosion $\left(\mathrm{N}_{\mathrm{e}}=10^{24} \mathrm{~cm}^{-3}, \mathrm{~T}_{\mathrm{e}}=10^{7} \mathrm{~K}\right)$, les profils expérimentaux ne permettent pas encore de valider la totalité de la chaîne de calcul nécessaire pour synthétiser un spectre de raies. Pour de tels plasmas, les profils de raies calculés au moyen du code sont confrontés à des simulations complètes de profils basées sur des méthodes de dynamique moléculaire que nous développons en parallèle.

Le programme fonctionne sur un mode interactif simple pour l'utilisateur. Il effectue les taches suivantes:

- lecture de la base de données de physique atomique et choix du 
système quantique optimal pour un domaine de fréquences donné. Les critères de choix dépendent des conditions de plasma et optimisent les couplages Stark induits par le microchamp entre les niveaux d'énergie de l'émetteur

- détermination des composantes de la transformée de Fourier de la fonction d'autocorrélation du moment dipolaire par diagonalisation de l'opérateur d'évolution pour une série pondérée de microchamps

- analyse de la structure inhomogène du profil, puis mélange des composantes pour tenir compte de l'effet de dynamique des ions. Le calcul du profil spectral par superposition des composantes est suivi de la prise en compte de l'effet Doppler par convolution.

Pour illustration, un cas simple est montré ci-dessous, accompagné des données statistiques du microchamp utiles pour le calcul.
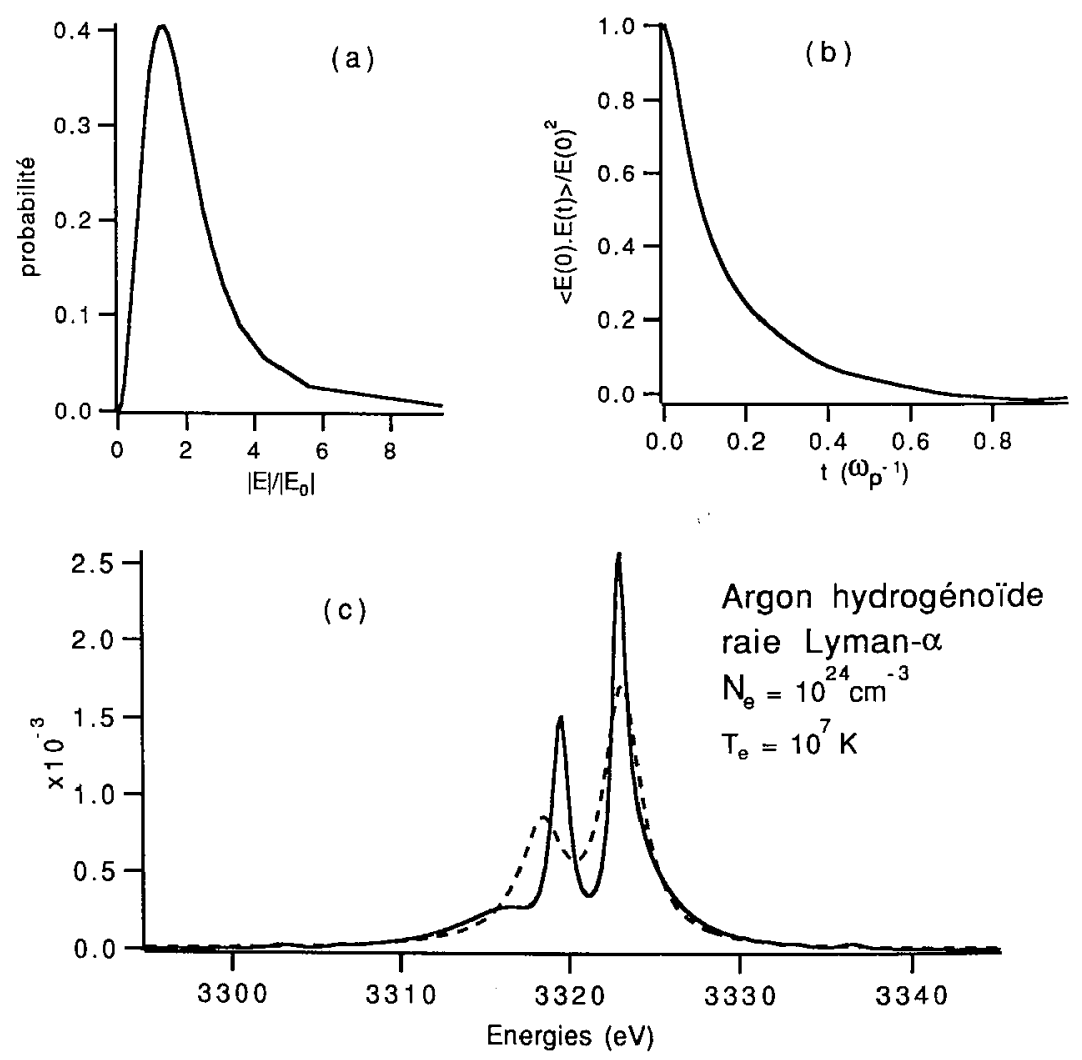

Ar ${ }^{17+} \mathrm{H}^{+}:$(a):fonction de distribution statique (APEX), (b):fonction d'autocorrélation du champ (simulation), (c):profils statique et dynamique (tiretés)

-A. Calisti, R.Stamm, B. Talin and R. W. Lee, Phys. Rev. A42, 5433 (1990)

-A. Calisti, F. Khelfaoui, R. Stamm and B. Talin in the Proceedings of the Conference on "Radiative Properties of Hot Dense Matter", ed. by W. Goldstein et al.(World Scientific, Hong Kong 1991) 